\title{
INDICE DE PRONTIDÃO A TECNOLOGIA: UM ESTUDO SOBRE AS DIMENSÕES POSITIVAS E NEGATIVAS EM RELAÇÃO AS FINTECHS
}

\author{
TECHNOLOGY READINESS INDEX: A STUDY ON \\ POSITIVE AND NEGATIVE DIMENSIONS IN RELATION \\ TO FINTECHS
}

Leandro Cearenço Limaa

Fabrício Zivianib

Fabio Corrêac

\begin{abstract}
RESUMO
Introdução: As novas possibilidades abertas pela tecnologia da informação e introduzidas pelas FinTechs, promovem, a evolução digital no segmento bancário, sobretudo, em plataformas digitais e de aplicações moveis. Objetivo: Analisar o índice de prontidão à tecnologia entre os usuários de Fintechs e verificar as dimensões positivas e negativas desta relação. Metodologia: A pesquisa tem natureza descritiva com abordagem quantitativa, em que, os testes foram aplicados a usuários de FinTechs via questionário para medir o índice de prontidão à tecnologia. Resultados: A rigor, foi possível analisar e confirmar a importância dos itens significativos para a formação do índice de prontidão dos usuários em relação às FinTechs. A pesquisa ressalta ainda que as dimensões positivas foram superiores às negativas nessa relação. Conclusões: Evidencia-se que os indivíduos são abertos e positivos em relação às FinTechs, o que denota alto índice de prontidão, pois em sua maioria são otimistas e inovativos. No entanto, quanto a dimensão negativa, conclui-se que ainda existe desconforto e insegurança em relação ao uso de novas tecnologias no segmento financeiro.
\end{abstract}

Descritores: Fintech, Tecnologia Financeira, Índice de Prontidão a Tecnologia.

\footnotetext{
a Mestre em Sistemas de Informação e Gestão do Conhecimento pela Universidade FUMEC. Consultor de negócios no Itaú-Unibanco S/A. E-mail: leandrolima.panamericano@gmail.com

b Doutor pelo Programa da Pós-Graduação em Ciência da Informação da Universidade Federal de Minas Gerais (UFMG). Diretor Executivo da TECINOVA Soluções Corporativas LTDA. Email: contato@fabricioziviani.com.br

c Doutor em Sistemas de Informação e Gestão do Conhecimento pela Universidade FUMEC. Email: fabiocontact@gmail.com
} 


\section{INTRODUÇÃO}

As empresas que prestam serviços financeiros em plataformas digitais e de aplicações móveis, denominadas FinTechs, vem auferindo atenção nos mercados mundiais nos últimos anos (DAPP, 2014; MCAULEY, 2015; CAHILL, 2015; GIBSON, 2015; KANTOX, 2016; MICU; MICU, 2016; TUCCI; GAUTSCHI; VISCUSI, 2016; WEN, 2016).

O mix de negócios e empresas estão se modificando nos países em função da tecnologia (TUCCI; GAUTSCHI; VISCUSI, 2016). Com isso, as novas possibilidades de envolvimento com o consumidor no segmento bancário, abertas pela Tecnologia da Informação ( $\mathrm{TI})$ e incorporadas por inovações via FinTechs, em geral promovem o surgimento e o crescimento de diversos negócios, como o empréstimo on-line (NORTHRUP; HANGEN; SWACK, 2016), que apresenta possibilidades que rompem limites, sobretudo, reduzem custos e barreiras geográficas.

Ao longo das últimas décadas, a evolução digital bancária começou nos terminais eletrônicos, passando por cartões inteligentes, chegando às plataformas digitais e dispositivos móveis. Para Wen (2016), cada um desses avanços foi significativo, com a popularização fortemente restringida pela aceitação dos usuários-alvo. Hoje, uma grande variedade de empresas FinTech envolvem os consumidores ao lançar soluções que facilitam pagamentos, transferências, empréstimos, financiamento de capital, plataformas de negociação, dentre outros (KANTOX, 2016).

Desde o surgimento das primeiras instituições financeiras até os dias atuais o objetivo principal no modelo de negócio bancário é a intermediação financeira entre indivíduos, grupos e organizações. Para Mota (2016, p.58) "entender o envolvimento do cliente com os produtos bancários torna-se chave para melhor direcionar esse mercado". O relatório da Corporação Interamericana de Investimentos de 2016 aponta que a expansão do uso de aplicativos desenvolvidos por empresas FinTech promovem simplicidade e velocidade nas interações em comparação com serviços in loco de bancos tradicionais (CAHILL, 2015). 
Nesse contexto, esta pesquisa tem como objetivo analisar o índice de prontidão dos usuários em relação as FinTechs e verificar as dimensões positivas e negativas dessa relação. Deste modo, este estudo apresenta relevância por verificar a propensão dos usuários em relação a adoção de inovações tecnológicas financeiras.

Para atingimento do referido objetivo, esta pesquisa se subdivide em seções. Além desta introdução, a seção seguinte apresenta o referencial teórico, contemplando as Fintechs e o índice de prontidão à tecnologia. Em sequência, os procedimentos metodológicos são explanados. Por conseguinte, os resultados advindos são apresentados e, posteriormente, as considerações finais são tecidas. Por fim, as referências bibliográficas, utilizadas ao longo desta pesquisa, são listadas e finalizam este estudo.

\section{REFERENCIAL TEÓRICO}

Esta seção destina-se a apresentar os fundamentos teóricos que norteiam o desenvolvimento desta pesquisa, iniciando pelas Fintechs.

\subsection{FINTECH}

De modo abrangente, o termo FinTech é definido por McAuley (2015) como um substantivo proveniente das palavras Finance Technology-Tecnologia Financeira em português. McAuley (2015) afirma se tratar de uma indústria econômica composta por empresas que utilizam a tecnologia para tornar os sistemas financeiros mais eficientes. Já para Dapp (2014) o termo FinTech refere-se às tecnologias modernas desenvolvidas para o setor financeiro bancário. No entanto, tal termo tem sido utilizado também para descrever a digitalização de produtos e serviços fornecidos por empresas que não são bancos.

Dapp (2014) aponta que FinTech é uma espécie de "movimento", que está sendo impulsionado por uma mudança estrutural digital no setor financeiro, dado pelo ritmo acelerado da evolução dos domínios de dispositivos móveis, pelos modernos métodos de análise de dados, pela personalização dos serviços on- 
line e pela crescente convergência das tecnologias de informação e comunicação.

Chuen e Teo (2015) referem-se a FinTech como serviços financeiros inovadores ou produtos entregues através de novas tecnologias. Já Micu e Micu (2016) apontam que FinTech representa um importante setor de serviços que se dedica a inovação nos serviços prestados às instituições financeiras, clientes e demais empresas que atuam nesse segmento.

Nesta pesquisa as FinTechs são caracterizadas como empresas que combinam modelos de negócios inovadores e tecnologia para permitir melhorar e provocar disrupção nos serviços financeiros bancários (GULAMHUSEINWALA; BULL; LEWIS, 2015).

Existem diversos tipos de FinTechs atuando no sistema financeiro brasileiro. As intermediações são as mais variadas e incluem ações como leilões, negociação, compra, venda, pagamentos, empréstimos, leasing, corretagem, câmbio, análise de risco, dentre outras (GIBSON, 2015). O tipo de FinTech mais recorrente no Brasil é o de pagamento móvel, ou seja, solução de pagamento sem dinheiro em espécie, nos quais, a intermediação é realizada através de dispositivos portáteis, tais como, smartphones ou outros dispositivos inteligentes sem fio, comumente usados no cotidiano da sociedade (GIBSON, 2015).

Para Dapp (2014) muito do que é hoje tecnologicamente possível, já podia ser visto em fascinantes filmes de ficção científica e ninguém pôde prever, com precisão, como seria o olhar do banco do futuro. No entanto, o autor alerta que estamos caminhando em uma direção altamente orientada para a internet e para o mundo virtual. $O$ que se aplica tanto à atividade bancária real quanto a orientação estratégica, no que diz respeito ao design de ramos de atuação, porque as possibilidades no setor financeiro se expandem ao longo do tempo e são multivariadas.

\section{2 ÍNDICE de Prontidão À TeCNOLOGIA}

O índice de prontidão à tecnologia TRI (Technology Readiness Index) diz respeito a propensão dos indivíduos em adotar inovações tecnológicas. Em 
outros termos, é a resultante de um conjunto de fatores que determinam a predisposição de um indivíduo para utilizar produtos ou serviços baseados em tecnologia (PARASURAMAN, 2000). Tais fatores de prontidão à tecnologia são representados, de acordo com Parasuraman e Colby (2001), por 4 diferentes aspectos, conforme representado na Figura 1.

Figura 1 - Modelo para índice de prontidão a tecnologias

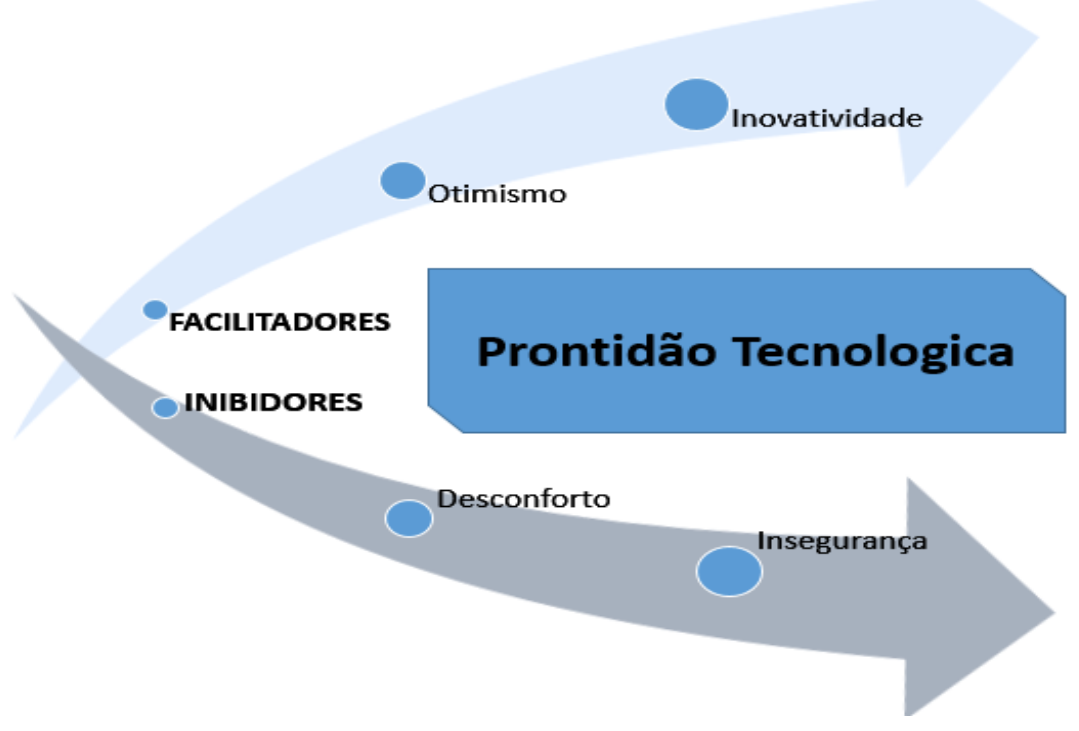

Fonte: Adaptado de Parasuraman e Colby (2001, tradução nossa)

$\mathrm{Na}$ ótica do TRI os fatores otimismo e inovatividade são facilitadores ou condutores da prontidão para o uso da tecnologia. Logo, são elementos positivos que representariam fatores motivacionais à adoção de novas tecnologias. Em contrapartida, os fatores desconforto e insegurança são limitadores ou inibidores negativos. Em consequência, seriam capazes de retardar, desmotivar ou impedir a adoção de novas tecnologias.

As dimensões facilitadoras e inibidoras da prontidão para o uso de novas tecnologias atuam de forma independente, sendo que uma pessoa pode apresentar combinações que motivam ou inibem simultaneamente. Esses fatores podem ser compreendidos como:

- Otimismo: dimensão positiva em relação à tecnologia e crença de que proporciona aos indivíduos maior controle, flexibilidade e eficiência nas suas vidas (PARASURAMAN; COLBY, 2001); 
- Inovatividade: dimensão positiva em relação à tecnologia que constitui tendência de o indivíduo ser pioneiro no uso ou adoção, bem como, ser líder ou formador de opinião a respeito de novas tecnologias (PARASURAMAN; COLBY, 2001);

- Desconforto: dimensão negativa e percepção de falta de controle sobre a tecnologia e o sentimento de ser pressionado ou oprimido por ela (PARASURAMAN; COLBY, 2001);

- Insegurança: dimensão negativa geradora de desconfiança da tecnologia e ceticismo em relação às próprias habilidades em utilizá-la de forma adequada (PARASURAMAN; COLBY, 2001).

Parasuraman (2000) assinala que as dimensões foram testados e classificados com escores. Desse modo, a prontidão geral para o uso de novos produtos ou serviços tecnológicos oferecidos ao consumidor é dada pela combinação desses quatro fatores e não pela capacidade técnica ou rapidez com que o indivíduo adota a tecnologia apresentada. Então, a classificação não se baseia apenas em uma das dimensões isoladamente, mas em aspectos relacionados aos quatro fatores que constituem o modelo.

O TRI de Parasuraman (2000) e Parasuraman e Colby (2001) é um instrumento que apresenta capacidade de predizer comportamentos quanto a adoção de novas tecnologias e, portanto, torna-se adequado ao intento desta pesquisa de analisar o índice de prontidão dos usuários em relação as FinTechs, considerando as dimensões positivas e negativas dessa relação.

\section{METODOLOGIA}

Esta pesquisa emprega a abordagem quantitativa por ser, segundo Moresi (2003), a mais apropriado para medir opiniões, atitudes, preferências e até mesmo comportamentos. Também é caracterizada como uma pesquisa empírica descritiva, de corte transversal, pois de acordo com Vergara (2014), a pesquisa descritiva capacita a exposição de características de determinada população ou fenômeno em dado momento histórico.

Como instrumento de coleta de dados utilizou-se o questionário com 36 
afirmativas do modelo TRI de Parasuraman e Colby (2001), fazendo uso da escala likert de 5 pontos e do tipo e-Survey (Quadro 1). Foram adicionadas 9 questões ao instrumento de pesquisa, referentes às caracterizações socioeconômicas e demográficas do indivíduo, sendo: (1) gênero, (2) idade; (3) estado civil; (4) escolaridade; (5) renda familiar; (6) Estado; (7) tipos de Fintech; (8) banco financeiro em que concentravam as transações; e (9) motivos para a escolha do banco. Assim, o instrumento de pesquisa é constituído por 45 variáveis.

\section{Quadro 1 - Variáveis de índice de prontidão a tecnologia do questionário}

\section{Dimensão/Variáveis}

\section{Otimismo}

OTI1. A tecnologia permite que as pessoas tenham mais controle sobre o seu dia-a-dia.

OTI2. Produtos e serviços que utilizam as mais novas tecnologias são muito mais convenientes de usar.

OTI3. Você gosta da ideia de fazer negócios pelo computador ou aplicativos, porque você não fica restrito ao horário comercial.

OTI4. Você prefere usar a tecnologia mais avançada disponível.

OTI5. Você gosta de aplicativos ou programas de computador que the permitam adequar as coisas às suas próprias necessidades.

OTI6. A tecnologia faz com que você fique mais eficiente no seu trabalho.

OTI7. Você considera as novas tecnologias mentalmente estimulantes.

OTI8. A tecnologia Ihe dá mais liberdade de movimento.

OTI9. Aprender sobre tecnologia pode ser tão compensador quanto a própria tecnologia.

OTI10. Você está seguro de que as maquinas seguirão suas instruções.

\section{Inovatividade}

INO1. Outras pessoas lhe pedem conselhos sobre novas tecnologias.

INO2. Parece que seus amigos estão aprendendo sobre as mais novas tecnologias mais do que você. ${ }^{*}$

INO3. Em geral, você está em os primeiros do seu grupo de amigos em adquirir uma nova tecnologia logo que ela surge.

INO4. Normalmente, você consegue entender os novos produtos e serviços de alta tecnologia sem ajuda de outros.

INO5. Você está atualizado com os últimos desenvolvimentos tecnológicos das suas áreas de interesse.

INO6. Você gosta do desafio de entender equipamentos de alta tecnologia.

INO7. Você tem menos problemas do que outras pessoas para fazer a tecnologia trabalhar para você.

Desconforto

DES1. Os serviços de suporte técnico (por telefone ou internet) não ajudam, porque não explicam as coisas em termos compreensíveis.

DES2. Às vezes, você acha que o sistema de tecnologia não são projetados para serem usados por pessoas comuns.

DES3. Não existe manual de produto ou serviço de alta tecnologia que seja escrito em uma linguagem simples. 
DES4. Quando você utiliza o suporte técnico de um fornecedor de produtos e serviços de alta tecnologia, às vezes você se sente como se alguém que sabe mais do que você estivesse tirando vantagem de você.

DES5. Na compra de um produto ou serviço de alta tecnologia, você prefere uma modelo básico a um modelo com muitas características adicionais.

DES6. E constrangedor quando você tem problemas com um equipamento de alta tecnologia, enquanto outras pessoas estão olhando.

DES7. Deveria haver cuidado ao substituir tarefas desempenhadas por pessoas pela tecnologia, pois novas tecnologias podem falhar.

DES8. Muitas das novas tecnologias apresentam riscos à saúde ou à segurança que não são descobertos até que as pessoas tenha utilizado a tecnologia.

DES9. Novas tecnologias tornam muito mais fácil para o governo e as empresas espionarem as pessoas.

DES10. As tecnologias parecem sempre falhar no pior momento possível.

\section{Insegurança}

INS1. Você não considera seguro fornecer o número do seu cartão de credito por aplicativos ou computadores.

INS2. Você não considera seguro fazer qualquer tipo de transação pela internet.

INS3. Você tem receio de que as informações que você envia pela internet são vistas por outras pessoas.

INS4. Você não se sente seguro em fazer negócios com uma empresa que só pode ser acessada pela internet.

INS5. Qualquer transação realizada eletronicamente deveria ser confirmada posteriormente por algo escrito.

INS6. Sempre que algo se torna automatizado, é necessário checar, cuidadosamente se a máquina ou computador não está cometendo erros.

INS7. O contato humano é muito importante quando se faz negócios com uma empresa.

INS8. Quando você liga para uma empresa, você prefere falar com uma pessoa do que com uma máquina.

INS9. Quando você fornece informação a uma máquina ou pela internet, você nunca pode ter certeza de que ela realmente chegou ao destino certo.

* indica score inverso

Fonte: Adaptado de Parasuraman e Colby (2001)

O endereço eletrônico do questionário foi encaminhado em aplicativos e redes sociais e fez uso da técnica Snowball, na qual os participantes iniciais compartilharam o instrumento de pesquisa a outros participantes de forma sucessiva. Para Baldin e Munhoz (2011, p. 50) essa técnica "é uma forma de amostra não probabilística [...] em que os participantes iniciais de um estudo indicam novos participantes que por sua vez indicam novos participantes e assim sucessivamente, até que seja alcançado o objetivo proposto".

Para análise dos dados foram utilizadas medidas de intervalos de confiança, desvio padrão e média (HAIR JUNIOR et. al., 2009) e a Análise Fatorial com o método de extração das componentes principais (MINGOTI, 2007). O software utilizado nas análises foi o R, versão 3.4.4. 


\section{APRESENTAÇÃO, ANÁLISE E DESCRIÇÃO DOS RESULTADOS}

Esta seção estabelece a apresentação, análise e descrição dos resultados da pesquisa, elaborada sobre os dados advindos de 306 indivíduos que responderam o questionário. Assim, esta seção é segmentada em subseções, a saber: análise descritiva da amostra (4.1), análise dos fatores (4.2) e analise fatorial exploratória (4.3).

\subsection{ANÁlise Descritiva da Amostra}

A amostra é composta por 306 indivíduos que responderam à pesquisa. Foi realizada análise dos outliers, que consiste em padrões de respostas diferente das demais (HAIR JUNIOR et al, 2009). Em outros termos, são avaliados comportamentos destoantes que sinalizam respostas descompromissadas. Após a análise, não foram identificados outliers. As variáveis constituintes da análise descritiva são apresentadas na Tabela 1.

Tabela 1 - Modelo para índice de prontidão a tecnologias

\begin{tabular}{|c|c|c|c|}
\hline Variáveis & & $\mathbf{N}$ & $\%$ \\
\hline \multirow{2}{*}{ Gênero } & Feminino & 128 & $41,8 \%$ \\
\hline & Masculino & 178 & $58,2 \%$ \\
\hline \multirow{4}{*}{ Idade } & De 18 a 24 & 39 & $12,7 \%$ \\
\hline & De 25 a 44 & 199 & $65,0 \%$ \\
\hline & De 45 a 59 & 64 & $20,9 \%$ \\
\hline & Acima de 60 & 4 & $1,3 \%$ \\
\hline \multirow{4}{*}{ Estado civil } & Casado & 183 & $59,8 \%$ \\
\hline & Divorciado & 15 & $4,9 \%$ \\
\hline & Solteiro & 103 & $33,7 \%$ \\
\hline & Viúvo & 5 & $1,6 \%$ \\
\hline \multirow{7}{*}{ Escolaridade } & Fundamental ( $\left.1^{\circ} \mathrm{Grau}\right)$ & 4 & $1,3 \%$ \\
\hline & Médio (2 Grau) & 99 & $32,4 \%$ \\
\hline & Superior completo & 113 & $36,9 \%$ \\
\hline & Especialização & 51 & $16,7 \%$ \\
\hline & Mestrado & 34 & $11,1 \%$ \\
\hline & Doutorado & 4 & $1,3 \%$ \\
\hline & Pós-doutorado & 1 & $0,3 \%$ \\
\hline \multirow{4}{*}{ Renda familiar } & Até $R \$ 1.874,00$ & 31 & $10,1 \%$ \\
\hline & De $R \$ 1.874,01$ a $R \$ 3.748,00$ & 88 & $28,8 \%$ \\
\hline & De $R \$ 3.748,01$ a $R \$ 9.370,00$ & 116 & $37,9 \%$ \\
\hline & De $R \$ 9.370,0$ a $R \$ 18.740,00$ & 49 & $16,0 \%$ \\
\hline
\end{tabular}

Inf. Inf., Londrina, v. 24, n. 2, p. 211 - 233, maio/ago. 2019. 


\begin{tabular}{llcc} 
& Acima de R\$ $18.740,01$ & 22 & $7,2 \%$ \\
\hline \multirow{2}{*}{ Estado } & Minas Gerais & 289 & $94,4 \%$ \\
& Outros & 17 & $5,6 \%$ \\
\hline
\end{tabular}

Fonte: Dados da pesquisa.

Os respondentes deveriam possuir faixa etária acima de 18 anos. Esse corte foi adotado considerando o critério de maioridade, que confere autonomia e discernimento da população em adotar a) produtos e serviços financeiros, b) produtos e serviços de inovação tecnológica. Ademais, os respondentes deveriam ser usuários de produtos ou serviços oferecidos por FinTechs, o que demanda faixa etária mínima de 18 anos.

Cabe ressaltar, ainda, que a amostra foi de composição não probabilística por conveniência, uma vez que nesse tipo de amostra a seleção dos respondentes é feita entre os que estão disponíveis para participar e que sejam capazes de fornecer as informações solicitadas, como apontam Hair Junior et al (2009) nas amostras não probabilísticas, a chance de seleção de um elemento da população é desconhecida.

Conforme apontado na Tabela 1, 58,2\% dos respondentes são do sexo masculino, enquanto $41,8 \%$ são do sexo feminino. A amostra apresentou maior concentração etária na faixa entre 25 a 44 anos de idade, compondo $65,0 \%$ dos respondentes, seguida da faixa de 45 a 59 anos foi de $20,9 \%$ da amostra. A faixa de 18 a 24 anos representou $12,7 \%$ e a menor concentração foi de respondentes a partir de 60 anos de idade $(1,3 \%)$.

Quanto ao estado civil ficou evidenciado que $59,8 \%$ dos respondentes eram casados enquanto $33,7 \%$ com menor concentração entre divorciados $4,9 \%$ e viúvos de 1,6\%. O grau de escolaridade dos respondentes aponta que a maioria possui de ensino superior completo a pós-doutorado $(66,30 \%)$, o que exprime autonomia e discernimento aos respondentes.

Em relação à renda familiar dos respondentes, 89,09\% possuem renda familiar acima de $\mathrm{R} \$ 1.874,01$. Quando perguntado o domicilio dos respondentes a pesquisa apontou que houve concentração no estado de Minas Gerais, onde $94,4 \%$ dos indivíduos residiam, e $5,6 \%$ dos respondentes mantinham domicilio em outros estados. 
Além das perguntas sócio econômicas e demográficas foi perguntado aos clientes quais os tipos de Fintechs utilizaram. Apesar de alguns indivíduos utilizarem mais de um tipo de Fintech, houve maior concentração no uso de Fintechs de pagamento que representou $71,2 \%$ da amostra, em seguida $48,7 \%$ dos respondentes apontaram que utilizam Fintechs de compra e $22,5 \%$ de venda, já as Fintechs para credito ou financiamento foram $20,6 \%$ dos respondentes da amostra.

Isso corrobora com a perspectiva de Gibson (2015), ao assinalar que o tipo de Fintech mais recorrente no Brasil é o de pagamento móvel, sendo a solução de pagamento sem dinheiro em espécie, nas quais, a intermediação é realizada através de dispositivos portáteis, tais como, smartphones ou outros dispositivos inteligentes sem fio, comumente usados no cotidiano da sociedade.

Quando se perguntou aos respondentes em relação ao banco em que concentravam as transações, $28,4 \%$ apontaram o Banco Itaú como preferência, já 19,9\% apontaram a Caixa Econômica Federal, 17,3\% apontaram o Banco do Brasil, 14,1\% concentram as transações no Bradesco, $14,1 \%$ concentram no Banco Santander, 0,3\% concentram no HSBC enquanto 5,9\% dos respondentes concentram as transações em outros bancos não especificados.

Também perguntou-se aos respondentes quanto aos motivos para a escolha do banco em que concentram as transações. O percentil de 43,8 alegaram ser a conta para o recebimento do salário o motivo principal, em seguida $37,9 \%$ apontaram os serviços oferecidos como motivo, 19,6\% afirmam se a localização, 19,3\% apontam as taxas praticadas como motivo de escolha, $17,6 \%$ apontaram a solidez do banco como motivo, $15,4 \%$ alegaram ser 0 atendimento personalizado, $14,1 \%$ afirmam que o fato de ser o mesmo banco de familiares e parceiros comerciais motiva a escolha 10,5\% apontaram a facilidade de acesso ao credito, 4,9\% apontaram que a indicação influência a escolha do banco e 19,3\% apontam outros motivos não especificados para escolher em qual banco concentra as transações. 


\subsection{Análise dos Fatores de Prontidão a Tecnologias}

As análises dos fatores de prontidão a tecnologias têm o objetivo de demonstrar as questões relacionadas a descrição dos constructos. Cabe ressaltar que a escala likert de concordância foi fixada para variar de 1 (Discordo Totalmente) a 5 (Concordo Totalmente). Dessa forma, intervalos estritamente menores que 3 indicam que os indivíduos tendem a discordar, enquanto que intervalos estritamente maiores que 3 que os indivíduos tendem a concordar e que intervalos que contém 3 indicam que não tenderam a discordar nem concordar. A Tabela 2 apresenta as variáveis relacionadas ao fator Otimismo.

Tabela 2 - Descrição das variáveis de caracterização do Otimismo

\begin{tabular}{lllll}
\hline Fator & & Média & D.P. & I.C. $-95 \%{ }^{1}$ \\
\hline \multirow{4}{*}{ Otimismo } & OTI1 & 4,06 & 0,86 & {$[3,95 ; 4,14]$} \\
& OTI2 & 3,97 & 0,89 & {$[3,87 ; 4,07]$} \\
& OTI3 & 4,30 & 0,92 & {$[4,19 ; 4,40]$} \\
& OTI4 & 3,81 & 1,14 & {$[3,68 ; 3,93]$} \\
& OTI5 & 4,28 & 0,89 & {$[4,18 ; 4,39]$} \\
& OTI6 & 4,28 & 0,86 & {$[4,18 ; 4,37]$} \\
& OTI7 & 3,62 & 1,06 & {$[3,50 ; 3,75]$} \\
& OTI8 & 4,12 & 0,97 & {$[4,00 ; 4,22]$} \\
& OTI9 & 3,71 & 1,11 & {$[3,58 ; 3,83]$} \\
& OTI10 & 3,58 & 1,07 & {$[3,46 ; 3,68]$} \\
\hline Intervalo Bootstrap & & &
\end{tabular}

Fonte: Dados da pesquisa.

No fator Otimismo (Tabela 2), os indivíduos tenderam a concordar com todas as afirmativas, uma vez que os intervalos de confiança não apresentam o valor inferior a 3. Além disso, os itens OTI3 ("Você gosta da ideia de fazer negócios pelo computador ou aplicativos porque você não fica restrito ao horário comercial"), OTI5 ("Você gosta de aplicativos ou programas de computador que Ihe permitam adequar as coisas às suas próprias necessidades") e OTI6 ("A tecnologia faz com que você fique mais eficiente no seu trabalho") apresentaram uma concordância significativamente maior que a dos demais, uma vez que seus intervalos não sobrepuseram os dos outros. 
Tabela 3 - Descrição das variáveis de caracterização da Inovatividade

\begin{tabular}{lllll}
\hline Fator & & Média & D.P. & I.C. - 95\% \\
\hline \multirow{4}{*}{ Inovatividade } & INO1 & 3,52 & 1,23 & {$[3,38 ; 3,65]$} \\
& INO2 & 2,94 & 1,20 & {$[2,79 ; 3,06]$} \\
& INO3 & 2,91 & 1,21 & {$[2,78 ; 3,06]$} \\
& INO4 & 3,66 & 1,13 & {$[3,54 ; 3,78]$} \\
& INO5 & 3,52 & 1,09 & {$[3,40 ; 3,63]$} \\
& INO6 & 3,63 & 1,15 & {$[3,50 ; 3,76]$} \\
INO7 & 3,77 & 1,06 & {$[3,64 ; 3,89]$} \\
\hline \multicolumn{1}{l}{ Intervalo Bootstrap } & & &
\end{tabular}

Fonte: Dados da pesquisa.

No fator Inovatividade (Tabela 3), os indivíduos tenderam a concordar com todas as afirmativas, com exceção das afirmativas INO2 ("Parece que seus amigos estão aprendendo sobre as mais novas tecnologias mais do que você") e INO3 ("Em geral, você está entre os primeiros do seu grupo de amigos em adquirir uma nova tecnologia logo que ela surge") em que os indivíduos tenderam a permanecer neutros.

Tabela 4 - Descrição das variáveis de caracterização do Desconforto

\begin{tabular}{llccl}
\hline Fator & & Média & D.P. & I.C. - 95\% ${ }^{\mathbf{1}}$ \\
\hline \multirow{7}{*}{ Desconforto } & DES1 & 2,91 & 1,20 & {$[2,78 ; 3,05]$} \\
& DES2 & 2,68 & 1,15 & {$[2,56 ; 2,81]$} \\
& DES3 & 2,64 & 1,21 & {$[2,51 ; 2,78]$} \\
& DES4 & 2,55 & 1,18 & {$[2,42 ; 2,69]$} \\
& DES5 & 2,78 & 1,22 & {$[2,64 ; 2,92]$} \\
& DES6 & 2,78 & 1,23 & {$[2,63 ; 2,92]$} \\
& DES7 & 3,45 & 1,07 & {$[3,33 ; 3,57]$} \\
& DES8 & 3,10 & 0,97 & {$[2,99 ; 3,20]$} \\
& DES9 & 4,04 & 0,96 & {$[3,94 ; 4,14]$} \\
& DES10 & 3,10 & 1,08 & {$[2,97 ; 3,22]$} \\
\hline
\end{tabular}

1 Intervalo Bootstrap

Fonte: Dados da pesquisa.

No fator Desconforto (Tabela 4), os indivíduos tenderam a discordar com a maioria dos itens, sendo que nos itens DES7 ("Deveria haver cuidado ao substituir tarefas desempenhadas por pessoas pela tecnologia, pois novas tecnologias podem falhar") e DES9 ("Novas tecnologias tornam muito mais fácil para o governo e as empresas espionarem as pessoas") houve tendência a concordar e nos itens DES1 ("Os serviços de suporte técnico (por telefone ou 
internet) não ajudam porque não explicam as coisas em termos compreensíveis"), DES8 ("Muitas das novas tecnologias apresentam riscos à saúde ou à segurança que não são descobertos até que as pessoas tenham utilizado a tecnologia") e DES10 ("As tecnologias parecem sempre falhar no pior momento possível") houve tendência a permanecer neutro.

Tabela 5 - Descrição das variáveis de caracterização da Insegurança

\begin{tabular}{llrll}
\hline Fator & & Média & D.P. & I.C. $-\mathbf{9 5 \%}{ }^{\mathbf{1}}$ \\
\hline \multirow{7}{*}{ Insegurança } & INS1 & 3,07 & 1,24 & {$[2,93 ; 3,20]$} \\
& INS2 & 2,81 & 1,30 & {$[2,68 ; 2,96]$} \\
& INS3 & 3,40 & 1,15 & {$[3,27 ; 3,53]$} \\
& INS4 & 2,85 & 1,31 & {$[2,70 ; 3,00]$} \\
& INS5 & 2,96 & 1,30 & {$[2,81 ; 3,10]$} \\
& INS6 & 3,67 & 1,09 & {$[3,55 ; 3,80]$} \\
& INS7 & 3,45 & 1,18 & {$[3,32 ; 3,59]$} \\
& INS8 & 3,84 & 1,14 & {$[3,71 ; 3,96]$} \\
& INS9 & 3,03 & 1,18 & {$[2,90 ; 3,16]$} \\
\hline 1 Intervalo Bootstrap & & &
\end{tabular}

Fonte: Dados da pesquisa.

No fator Insegurança (Tabela 5), os indivíduos tenderam a concordar com os itens INS3 ("Você tem receio de que as informações que você envia pela internet são vistas por outras pessoas"), INS6 ("Sempre que algo se torna automatizado é necessário checar, cuidadosamente, se a máquina ou computador não está cometendo erros"), INS7 ("O contato humano é muito importante quando se faz negócios com uma empresa") e INS8 ("Quando você liga para uma empresa, você prefere falar com uma pessoa do que com uma máquina"), a permanecer neutros com os itens INS1 ("Você não considera seguro fornecer o número do seu cartão de credito por aplicativos ou computadores"), INS4 ("Você não se sente seguro em fazer negócios com uma empresa que só pode ser acessada pela internet"), INS5 ("Qualquer transação realizada eletronicamente deveria ser confirmada posteriormente por algo escrito") e INS9 ("Quando você fornece informação a uma máquina ou pela internet, você nunca pode ter certeza de que ela realmente chegou ao destino certo") e a discordar do item INS2 ("Você não considera seguro fazer qualquer tipo de transação pela internet"). 


\subsection{ANÁlise FATORIAL EXPLORATÓRIA}

$\mathrm{Na}$ análise fatorial exploratória, a abordagem foi ajustada ao modelo de pesquisa proposto apresentado na Figura 1 em que o constructo FinTech que representa a "tecnologia" era de segunda ordem, ou seja, não era formado diretamente pelos itens "perguntas". Para tratar essa característica da estrutura de mensuração, foi utilizada a abordagem "Two-Step" (SANCHES, 2013). Desse modo, primeiramente foram computados os escores das variáveis latentes de primeira ordem, utilizando a Análise Fatorial com o método de extração das componentes principais (MINGOTI, 2007).

Para analisar a qualidade e validade dos constructos de primeira ordem foi verificada a dimensionalidade, confiabilidade e validade convergente. Para verificar a validade convergente foi utilizado o critério proposto por Fornell e Larcker (1981), que indica validação convergente quando a Variância Média Extraída - AVE for superior a 50\% (HENSELER; RINGLE; SINKOVICS, 2009) ou $40 \%$ no caso de pesquisas exploratórias (NUNNALY; BERNSTEIN, 1994).

Para mensurar a confiabilidade foi utilizado o Alfa de Cronbach (AC) e a Confiabilidade Composta (CC) (CHIN, 1998). De acordo com Tenenhaus et al (2005) os indicadores AC e CC devem ser maiores que 0,70 para uma indicação de confiabilidade do constructo, sendo que em pesquisas exploratórias valores acima de 0,60 também são aceitos (HAIR JUNIOR et al, 2009).

Para verificar a dimensionalidade dos constructos foi utilizado o critério das retas paralelas (HOYLE; DUVALL, 2004) que retorna a quantidade de dimensões do constructo. A adequação da amostra para utilização da análise fatorial foi medida através do indicador KMO que indica a proporção da variância dos dados que pode ser considerada comum a todas as variáveis. É uma medida que varia de 0,0 a 1,0, sendo que quanto mais próximo de 1,0 (unidade) mais apropriada será a amostra à aplicação da análise fatorial. É adequado aplicar a Análise Fatorial Exploratória ao conjunto de variáveis quando o $\mathrm{KMO}$ for maior que 0,50 .

A Tabela 6 apresenta a Análise Fatorial inicial e final. 
Tabela 6 - Análise fatorial dos constructos

\begin{tabular}{|c|c|c|c|c|c|c|c|}
\hline \multirow{2}{*}{ Fator } & & \multicolumn{3}{|c|}{ Modelo Inicial } & \multicolumn{3}{|c|}{ Modelo Final } \\
\hline & & C.F. ${ }^{1}$ & Com. $^{2}$ & Peso & C.F. ${ }^{1}$ & Com. $^{2}$ & Peso \\
\hline \multirow{10}{*}{ Otimismo } & OTI1 & 0,767 & 0,589 & 0,127 & 0,767 & 0,589 & 0,127 \\
\hline & OTI2 & 0,802 & 0,643 & 0,132 & 0,802 & 0,643 & 0,132 \\
\hline & OTI3 & 0,732 & 0,536 & 0,121 & 0,732 & 0,536 & 0,121 \\
\hline & OTI4 & 0,793 & 0,629 & 0,131 & 0,793 & 0,629 & 0,131 \\
\hline & OTI5 & 0,832 & 0,692 & 0,137 & 0,832 & 0,692 & 0,137 \\
\hline & OTI6 & 0,726 & 0,527 & 0,120 & 0,726 & 0,527 & 0,120 \\
\hline & OTI7 & 0,835 & 0,697 & 0,138 & 0,835 & 0,697 & 0,138 \\
\hline & OTI8 & 0,747 & 0,558 & 0,123 & 0,747 & 0,558 & 0,123 \\
\hline & OTI9 & 0,757 & 0,574 & 0,125 & 0,757 & 0,574 & 0,125 \\
\hline & OTI10 & 0,781 & 0,610 & 0,129 & 0,781 & 0,610 & 0,129 \\
\hline \multirow{7}{*}{ Inovatividade } & INO1 & 0,829 & 0,688 & 0,189 & 0,829 & 0,688 & 0,189 \\
\hline & INO2i & 0,520 & 0,271 & 0,119 & 0,520 & 0,271 & 0,119 \\
\hline & INO3 & 0,784 & 0,615 & 0,179 & 0,784 & 0,615 & 0,179 \\
\hline & INO4 & 0,877 & 0,770 & 0,200 & 0,877 & 0,770 & 0,200 \\
\hline & INO5 & 0,873 & 0,762 & 0,199 & 0,873 & 0,762 & 0,199 \\
\hline & INO6 & 0,819 & 0,672 & 0,187 & 0,819 & 0,672 & 0,187 \\
\hline & INO7 & 0,781 & 0,609 & 0,178 & 0,781 & 0,609 & 0,178 \\
\hline \multirow{10}{*}{ Desconforto } & DES1 & 0,692 & 0,479 & 0,153 & 0,700 & 0,490 & 0,156 \\
\hline & DES2 & 0,802 & 0,643 & 0,178 & 0,806 & 0,650 & 0,180 \\
\hline & DES3 & 0,798 & 0,637 & 0,177 & 0,804 & 0,646 & 0,180 \\
\hline & DES4 & 0,773 & 0,598 & 0,171 & 0,783 & 0,613 & 0,175 \\
\hline & DES5 & 0,654 & 0,427 & 0,145 & 0,658 & 0,433 & 0,147 \\
\hline & DES6 & 0,630 & 0,397 & 0,140 & 0,627 & 0,393 & 0,140 \\
\hline & DES7 & 0,663 & 0,440 & 0,147 & 0,653 & 0,426 & 0,146 \\
\hline & DES8 & 0,582 & 0,338 & 0,129 & 0,567 & 0,321 & 0,127 \\
\hline & DES9 & 0,226 & 0,051 & 0,050 & & & \\
\hline & DES10 & 0,710 & 0,504 & 0,157 & 0,707 & 0,500 & 0,158 \\
\hline \multirow{9}{*}{ Insegurança } & INS1 & 0,844 & 0,712 & 0,168 & 0,844 & 0,712 & 0,168 \\
\hline & INS2 & 0,742 & 0,550 & 0,147 & 0,742 & 0,550 & 0,147 \\
\hline & INS3 & 0,801 & 0,642 & 0,159 & 0,801 & 0,642 & 0,159 \\
\hline & INS4 & 0,836 & 0,699 & 0,166 & 0,836 & 0,699 & 0,166 \\
\hline & INS5 & 0,778 & 0,605 & 0,155 & 0,778 & 0,605 & 0,155 \\
\hline & INS6 & 0,646 & 0,418 & 0,129 & 0,646 & 0,418 & 0,129 \\
\hline & INS7 & 0,703 & 0,494 & 0,140 & 0,703 & 0,494 & 0,140 \\
\hline & INS8 & 0,610 & 0,373 & 0,121 & 0,610 & 0,373 & 0,121 \\
\hline & INS9 & 0,733 & 0,537 & 0,146 & 0,733 & 0,537 & 0,146 \\
\hline
\end{tabular}

${ }^{1}$ Carga fatorial; ${ }^{2}$ Comunalidade.

Fonte: Dados da pesquisa.

Ressalta-se que o item INO2 ("Parece que seus amigos estão aprendendo sobre as mais novas tecnologias mais do que você") foi invertido devido ao fato de estar em um sentido contrário ao dos demais itens do constructo, passando 
a ser denominado como INO2i ("Parece que seus amigos não estão aprendendo sobre as mais novas tecnologias mais do que você"). Logo, pode-se observar que o item DES9 ("Novas tecnologias tornam muito mais fácil para o governo e as empresas espionarem as pessoas") do constructo Desconforto foi retirado do modelo devido ao fato de ter apresentado uma carga fatorial menor que 0,50 . No modelo final, todos os itens apresentaram cargas fatoriais satisfatórias.

A Tabela 7 apresenta os resultados para a validade e qualidade dos fatores. Logo, pode-se destacar que: a) todos os fatores apresentaram validação convergente (AVE > 0,50). b) Todos os fatores apresentaram Alfa de Cronbach (AC) e Confiabilidade Composta (CC) acima de 0,60 , logo todos os constructos apresentaram os níveis exigidos de confiabilidade.

Em todos os fatores o ajuste da Análise Fatorial foi adequado, uma vez que todos os $\mathrm{KMO}$ foram maiores ou iguais a 0,50 e todos os construtos foram unidimensionais segundo o critério de retas paralelas.

Tabela 7 - Validação dos fatores

\begin{tabular}{lcccccc}
\hline Fator & Itens & AVE $^{\mathbf{1}}$ & $\mathbf{A C}^{\mathbf{2}}$ & $\mathbf{C C}^{\mathbf{3}}$ & $\mathbf{K M O}^{\mathbf{4}}$ & Dim. $^{\mathbf{5}}$ \\
\hline Otimismo & 10 & 0,605 & 0,926 & 0,906 & 0,928 & 1 \\
Inovatividade & 7 & 0,627 & 0,894 & 0,881 & 0,903 & 1 \\
Desconforto & 9 & 0,497 & 0,871 & 0,856 & 0,889 & 1 \\
Insegurança & 9 & 0,559 & 0,900 & 0,881 & 0,908 & 1 \\
\hline
\end{tabular}

${ }^{1}$ Variância Extraída; ${ }^{2}$ Alfa de Cronbach; ${ }^{3}$ Confiabilidade Composta; 4 Medida de adequação da amostra de Kaiser-Meyer-Olkin; ${ }^{5}$ Dimensionalidade.

Fonte: Dados da pesquisa.

Embora os fatores Otimismo, Inovatividade, Desconforto e Insegurança (constructos de primeira ordem) sejam reflexivos, o constructo FinTech (constructo de segunda ordem) é formativo. Dessa forma os constructos de primeira ordem são as causas do constructo de segunda ordem, enquanto que os itens (perguntas) são os reflexos de seus respectivos constructos de primeira ordem. A validação de um modelo estrutural formativo requer diferentes abordagens do modelo reflexivo. A avaliação convencional de validação e confiabilidade dos constructos não devem ser aplicados nos modelos formativos (BOLLEN, 1989). Portanto, para avaliar o modelo formativo foi verificado se os 
pesos eram significativos ou maiores que 0,20 e se as cargas fatoriais eram maiores que 0,60 (CHIN, 1998). Também foi avaliado se os Fatores de Inflação da Variância (VIF) eram maiores que 5, evitando assim problemas de multicolinearidade.

Já para os constructos reflexivos, os itens com cargas fatoriais menores que 0,50 devem ser eliminados (HAIR JUNIOR et al, 2009), pois ao não contribuir de forma relevante para formação da variável latente, prejudicam o alcance das suposições básicas para validade e qualidade dos indicadores criados para representar o conceito de interesse. Para de testar a validade convergente desses construtos foi utilizado novamente o critério proposto por Fornell e Larcker (1981). Para mensurar a confiabilidade dos constructos foram utilizados novamente os indicadores Alfa de Cronbach (A.C.) e Confiabilidade Composta (C.C.). Para a validade discriminante foi utilizado o critério de Fornell e Larcker (1981), que garante a validade discriminante quando a variância extraída (AVE) de um constructo for maior que a variância compartilhada desse constructo com os demais. Além disso, para verificar a dimensionalidade dos constructos foi utilizado novamente o critério das retas paralelas.

A Tabela 8 apresenta os pesos com seus respectivos valores-p, as cargas fatoriais, comunalidades e o Fator de Inflação da Variância (VIF) para os constructos formativos.

Tabela 8 - Validação dos constructos

\begin{tabular}{llllllll}
\hline Constructos & & Peso & Valor-p & I.C. $-95 \%$ & C.F. $^{\mathbf{2}}$ & Com. $^{\mathbf{3}}$ & VIF \\
\hline \multirow{4}{*}{ FinTech } & Otimismo & 0,751 & 0,000 & {$[0,58 ; 0,90]$} & 0,973 & 0,947 & 2,427 \\
& Inovatividade & 0,213 & 0,046 & {$[0,01 ; 0,42]$} & 0,854 & 0,729 & 2,569 \\
& Conforto & $-0,085$ & 0,177 & {$[-0,20 ; 0,04]$} & 0,277 & 0,077 & 1,876 \\
& Segurança & 0,216 & 0,014 & {$[0,05 ; 0,39]$} & 0,515 & 0,265 & 2,103 \\
\hline
\end{tabular}

${ }^{1}$ Intervalo Bootstrap; ${ }^{2}$ Carga fatorial; ${ }^{3}$ Comunalidade.

Fonte: Dados da pesquisa.

Cabe ressaltar que os indicadores Desconforto e Insegurança foram invertidos, uma vez que estavam em um sentido contrário ao dos indicadores Otimismo e Inovatividade. Dessa forma, estes indicadores passaram a ser denominados Conforto e Segurança.

Além disso, tem-se que: com relação ao constructo formativo; a maioria 
dos itens apresentou peso significativo, com exceção do item Conforto. Dessa forma, foi possível confirmar a importância dos itens significativos para a formação do indicador FinTech. As cargas fatoriais de Otimismo e Inovatividade foram superiores a 0,60. A estatística VIF foi menor que $5 \mathrm{em}$ todos os itens, indicando assim ausência de problemas de multicolinearidade.

\section{CONSIDERAÇÕES FINAIS}

Ao identificar os fatores que influenciam a prontidão às plataformas FinTech, buscou-se apoio na aplicabilidade da escala TRI, que propôs analisar o índice de prontidão às novas tecnologias. A pesquisa apontou que as afirmações em todas as dimensões foram positivas e denotam alto índice de prontidão dos usuários em relação às FinTechs.

Cabe ressaltar ainda que, ficou evidenciado que os respondentes são positivos, isso porque, a pesquisa aponta que são abertos e propensos, pois, em sua maioria são otimistas e inovativos em relação às FinTechs. No entanto a pesquisa aponta também que os respondentes se sentem desconfortáveis e inseguros em relação ao uso das novas tecnologias no segmento financeiro, parte pela complexidade das novas tecnologias e parte pelo baixo nível de compreensão e conhecimento das plataformas disponíveis no mercado.

Acredita-se que o presente estudo acarretará importantes contribuições capazes de nortear as startups e as empresas que atuam no sistema financeiro brasileiro, sobretudo, no segmento bancário, de modo que possam redefinir e direcionar melhor as estratégias no mercado. Além de contribuir para a academia em futuros estudos que envolvam a temática.

A limitação deste estudo refere-se à dificuldade de se alcançar maior número de respondentes de outras unidades federativas do Brasil, pois a técnica de Snowball, por se utilizar de redes de relacionamento virtuais acaba sendo limitada a bolhas de concentração regionais.

Como pesquisa futura, sugere-se uma técnica de pesquisa que de maior alcance regional no país a fim de se refletir melhor e otimizar a capacidade preditiva para cada grupo detectado. 


\section{REFERÊNCIAS}

BALDIN, N.; MUNHOZ, E. M. B. Educação ambiental comunitária: uma experiência com a técnica de pesquisa snowball (bola de neve). Revista Eletrônica do Mestrado em Educação Ambiental, v. 27, 2011, p. 46-60.

BOLLEN, K. A. A new incremental fit index for general structural equation models. Sociological Methods \& Research, v. 17, n. 3, 1989, p. 303-316. DOI doi.org/10.1177/0049124189017003004

CAHILL, Richard. Money \& Change: How FinTech startups and alternative banking companies are redefining finance through design, Digital Iteraction Design, University of Dundee, Dundee, 2015. Disponível em: http://lab.rc3.me/deliverables/RCahill-SocialMobileApps-MoneyAndChange.pdf Acesso em: 03 nov. 2017.

CHIN, W. W. The partial least squares approach to structural equation modeling. In: MARCOULIDES, G. A. Modern methods for business research, New York: Psichology Press, 1998, p. 295-336.

CHUEN, David L. K.; TEO, E. G. S. Emergence of FinTech and the LASIC principles. The Journal of Financial Perspectives, v. 3, n. 3, 2015.

DAPP, T. F. Fintech - the digital (r)evolution in the financial sector. Frankfurt, Deutsche Bank Research, 2014. Disponível em:

http://www.dbresearch.com/PROD/RPS EN-

PROD/PROD0000000000451941/Fintech_\%E2\%80\%93_The_digital_\%28r\%2 9evolution_in_the_financia.pdf. Acesso em: 04 set. 2018.

FORNELL, C.; LARCKER, D. F. Evaluating structural equation models with unobservable variables and measurement error. Journal of marketing research, v. 18, n. 1, 1981, p. 39-50.

GIBSON, J. The impact FinTech is having on the financial services industry in Ireland. 2015. 165f. Dissertação (Mestrado em Administração) Dublin Business School, Irlanda, 2015.

GULAMHUSEINWALA, I.; BULL, T.; LEWIS, S. FinTech is gaining traction and young, high-income users are the early adopters. Journal of Financial

Perspectives: FinTech. v.3, n.3, 2015, p.16-23.

HAIR JUNIOR, J. F; BLACK, B. J.; ANDERSON, R. E.; TATHAM, R. L. Análise multivariada de dados. Porto Alegre: Bookman, 2009.

HENSELER, J.; RINGLE, C. M.; SINKOVICS, R. R. The use of partial least squares path modeling in international marketing. Advances in International Marketing, v. 20, 2009, p. 277-319. 
HOYLE, R. H.; DUVALL, J. L. Determining the number of factors in exploratory and confirmatory factor analysis. In: KAPLAN, D. (Ed.): The SAGE hand book of quantitative methodology for the social sciences. Thousand Oaks: Sage. 2004, p. 301-315.

KANTOX. "Fintech" definition, KantoxGlossary, 2016. Disponível em: http://kantox.com/en/glossary/fintech. Acesso em 20 jul. 2018.

MCAULEY, D. What is FinTech? 2015. Disponível em: https://medium.com/wharton-fintech/what-is-fintech-77d3d5a3e677\#.irb06o6mv Acesso em: 20 jan. 2018.

MICU, I.; MICU, A. Financial technology (FinTech) and its implementation on the romanian non-banking capital market. Pratical Applications Science, Bucareste, v. 4, n. 2, 2016, p. 379-384.

MINGOTI, S. A. Análise de dados através de Métodos de Estatística Multivariada: uma abordagem aplicada. Belo Horizonte: UFMG, 2007.

MORESI, E. (Org). Manual de Metodologia da Pesquisa. Universidade Católica de Brasília: Distrito Federal, 2003.

MOTA, Karla Roberta Revert. Envolvimento do consumidor com produtos e serviços bancários. 2016. 165f. Dissertação (Mestrado em Administração) Universidade FUMEC, Belo Horizonte, 2016.

NORTHRUP, J.; HANGEN, E.; SWACK, M. CDFIs and online business lending: a review of recent progress challenges, and opportunities. The Carsey School of Public Policy, New Hampshire, 2016.Disponível em: https://scholars.unh.edu/cgi/viewcontent.cgi?article=1286\&context=carsey. Acesso em: 17 mar. 2018.

NUNNALY, J.; BERNSTEIN, I. Psychometric Theory. McGraw-Hill: New York. 1994.

PARASURAMAN, A. Technology Readiness Index (TRI): a multiple-item scale to measure readiness to embrace new technologies. Journal of Service Research, v. 2, n. 4, 2000, p. 307-320.

PARASURAMAN, A.; COLBY, C. Techno-ready marketing: how and why your customers adopt technology. New York: The Free Press, 2001.

SANCHEZ, G. PLS Path Modeling with R. Berkeley: Trowchez Editions, 2013.

TENENHAUS, M.; VINZI, V. E.; CHATELIN, Y.; LAURO, C. PLS path modeling. Computational Statistics \& Data Analysis, v. 48, 2005, p. 159-205.

TUCCI, Christopher L.; GAUTSCHI, Heidi; VISCUSI, Gianluigi. Switzerland's digital furniture: facts challenges recommendations. EPFL - College of 
Management of Technology, Lausanne, Suíça, 2016. Disponível em: https://www.six-group.com/dam/downloads/epfl-study-switzerland-digitalfuture.pdf. Acesso em: 16 mar. 2017.

VERGARA, Sylvia Constant. Projetos e Relatórios de Pesquisa em Administração. São Paulo: Atlas S.A. 2014.

WEN, C. FinTech acceptance research in Finland: case Company Plastc. 2016. 79f. Dissertação (Mestrado em Ciências Econômicas e Administração de Negócios) - Aalto University School of Business, Finlândia, 2016.

\title{
TECHNOLOGY READINESS INDEX: A STUDY ON POSITIVE AND NEGATIVE DIMENSIONS IN RELATION TO FINTECHS
}

\begin{abstract}
Introduction: The new possibilities opened by information technology and introduced by FinTechs, promote, the digital evolution in the banking segment, mainly in digital platforms and mobile applications. Objective: To analyze the technology readiness index among Fintechs users and to verify the positive and negative dimensions of this relationship. Methodology: The research has a descriptive nature with quantitative approach, in which, the tests were applied to FinTechs users via questionnaire to measure the readiness index to the technology. Results: Strictly speaking, it was possible to analyze and confirm the importance of significant items for the formation of the user readiness index in relation to FinTechs. The research also emphasizes that the positive dimensions were superior to the negative ones in this relation. Conclusions: Show that individuals are open and positive about FinTechs, which indicates a high level of readiness, since most are optimistic and innovative. However, regarding the negative dimension, we can conclude that there is still discomfort and insecurity regarding the use of new technologies in the financial segment.
\end{abstract}

Descriptors: Fintech, Financial Technology, Technology Readiness Index.

\section{INDICE DE PRONTITUD LA TECNOLOGÍA: UN ESTUDIO SOBRE LAS DIMENSIONES POSITIVAS Y NEGATIVAS EN RELACIÓN CON FINTECHS}

\begin{abstract}
RESUMEN
Introducción: Las nuevas posibilidades abiertas por la tecnología de la información e introducidas por las FinTechs, promueven, la evolución digital en el segmento bancario, sobre todo, en plataformas digitales y de aplicaciones móviles. Objetivo: Analizar el índice de prontitud a la tecnología entre los usuarios de Fintechs y verificar las dimensiones positivas y negativas de esta relación. Metodología: La investigación tiene carácter descriptivo con abordaje cuantitativo, en el cual, las pruebas fueron aplicadas a usuarios de FinTechs vía cuestionario para medir el índice de prontitud a la tecnología.
\end{abstract}

Inf. Inf., Londrina, v. 24, n. 2, p. 211 - 233, maio/ago. 2019. 
Resultados: En rigor, fue posible analizar y confirmar la importancia de los elementos significativos para la formación del índice de prontitud de los usuarios en relación a las FinTech. La investigación resalta además que las dimensiones positivas fueron superiores a las negativas en esa relación. Conclusiones: Evidencia que los individuos son abiertos y positivos en relación a las FinTechs, lo que denota alto índice de prontitud, pues en su mayoría son optimistas e innovadores. Sin embargo, en cuanto a la dimensión negativa, podemos concluir que todavía existe incomodidad e inseguridad en relación al uso de nuevas tecnologías en el segmento financiero.

Descriptores: Fintech, Tecnología Financiera, Índice de Prontitud a la Tecnología. 